\title{
Graph Signal Processing Based Cross-Subject Mental Task Classification Using Multi-Channel EEG Signals
}

This paper was downloaded from TechRxiv (https://www.techrxiv.org).

\section{LICENSE}

CC BY 4.0

SUBMISSION DATE / POSTED DATE

26-02-2022 / 03-03-2022

\section{CITATION}

mathur, priyanka; Chakka, Vijay Kumar (2022): Graph Signal Processing Based Cross-Subject Mental Task Classification Using Multi-Channel EEG Signals. TechRxiv. Preprint.

https://doi.org/10.36227/techrxiv.19242750.v1

$\mathrm{DOI}$

10.36227/techrxiv.19242750.v1 


\title{
Graph Signal Processing Based Cross-Subject Mental Task Classification Using Multi-Channel EEG Signals
}

\author{
Priyanka Mathur, Student Member, IEEE, and Vijay Kumar Chakka, Senior Member, IEEE
}

\begin{abstract}
Classification of mental tasks from electroencephalogram (EEG) signals play a crucial role in designing various brain-computer interface (BCl) applications. Most of the current techniques consider each channel as independent, neglecting the functional connectivity of the brain during mental activity and are primarily subject specific. This paper proposes a graph signal representation to classify a pair of mental tasks using multichannel EEG signals (MTMC-EEG) with cross subject classification within the database. Here, each channel of EEG signal corresponds to nodes of the task based graph whose EEG time series resides on the respective nodes. Functional connectivity of the brain between these nodes is obtained using smoothness constraint based Graph Signal Processing (GSP) technique. Graph spectral features namely, two-norm total variation of eigen vector (TNTV) corresponding to weighted adjacency matrix, graph Laplacian energy (GLE) using eigenvalues of Laplacian matrix and convex sum of TNTV and GLE in the form of joint total variation energy (JTVE) are proposed in this paper. The performance of the proposed methodology is evaluated on publicly available two different databases of MTMC EEG signals using benchmark classifiers and compared with the state of the art. Further, the superiority of the proposed metric obtained from the smoothened graph of GSP technique is validated by comparing it with Pearson correlation and Gaussian radial basis function (RBF) based functional connectivity in terms of accuracy, F-Score, and information transfer rate (ITR). The robustness of the proposed method is validated by adding white Gaussian noise (AWGN) to the EEG signals using different SNRs.
\end{abstract}

Index Terms-Brain-Computer Interface (BCI), MTMC EEG Signals, Graph Signal Processing (GSP), Laplacian matrix,two norm total variation of eigen vector (TNTV), graph Laplacian energy (GLE), joint total variation energy (JTVE) and cross subject classification.

\section{INTRODUCTION}

$\mathbf{M}$ ENTAL task based multi channel EEG signals (MTMC- EEG) represent the state of the brain while performing the specific tasks like imagining counting letters, arithmetic multiplication, subtraction, etc. The classification of these mental tasks is useful in building a neuro feedback system that finds a vital role in Brain Computer Interface (BCI) applications [1]. Several techniques have been reported in the literature to extract distinctive features from MTMC-EEG signals for the classification of mental based tasks which serves as input to a classifier like Support Vector Machine (SVM), K-Nearest Neighbour (KNN), linear discriminant classifier (LDA) etc. [2]. These multi-electrode EEG signals captures the brain's electrical activity during mental tasks along both spatial and temporal directions. The researchers have mostly utilised the temporal variations by extracting several feature extraction methods like entropy, cross-correlation [3], energy, and power spectral density [4]-[6]. Signal decomposition

Priyanka Mathur, Vijay Kumar Chakka are with Department of Electrical Engineering, Shiv Nadar University, Greater Noida. E-mail: pm133@snu.edu.in, vijay.chakka@snu.edu.in methods using discrete wavelet transform (DWT), empirical mode decomposition(EMD), multivariate empirical wavelet transform are also explored by the researchers [7]-[12] in extracting features for mental task classifications. Although these methods can provide satisfactory performance, they do not provide any solution for selecting a suitable number of signal decomposition levels [13]. Recently, deep learning methods like Convolution Neural Network, long short-term memory (LSTM) were proposed by authors [14], [15] combines feature extraction and classification in one architecture requiring large sized GPU.

The existing literature for mental task EEG signal classification mostly relied on features derived from individual channels rather than EEG-based functional connectivity. Each channel was considered as an independent signal thereby giving a high dimensional feature vector combined from all spatial channels. To reduce the dimensionality of the features from multi-channel EEG signals, authors in [16], [17] have applied various channel selection techniques together with signal decomposition to obtain features for classification. However, the functional connectivity-based methods can provide meaningful interpretation for the analysis of brain functions. For instance, 
authors in [18], [19], have analysed brain imaging signals using functional connections among brain areas for identifying Alzheimer's disease and fingerprinting respectively. EEG based functional connections are exploited by authors in [20], [21] for workload detection and dimensionality reduction.

Generally, learning a connectivity graph from the given signals include calculating statistical based similarity function like Gaussian radial basis function (RBF) or Pearson correlation which are purely based on the observations [22]. These methods are noise sensitive and require a large amount of observations and may be negative. Obtaining the graph topology using Graph Signal Processing (GSP) based learning ensures non-negativity of the adjacency matrix. Prior information of MTMC EEG signal classification [9] can also be utilised using GSP by embedding smoothness of signal variation between nodes and bandlimitedness in the graph spectral domain etc. [23], [24]

Motivated by this, learning the mental task based functional brain connectivity from multi channel EEG signal using GSP technique is addressed in this paper. We propose graph signal representation of EEG signals corresponding to each task, where each channel corresponds to the nodes of the graph representing the different regions of the brain and the observations over all channels correspond to the graph signal. Eigen decomposition of the weighted adjacency matrix and Laplacian matrix of the graph is used to extract spectral features. The average of the two norm total variation of eigen vectors (TNTV) belonging only to lower spectral content [25] is computed as a feature from weighted adjacency matrix and eigen values corresponding to Laplacian matrix is used to obtain feature metric of graph Laplacian energy (GLE). A convex sum metric combining the ability of both TNTV and GLE is taken in the form of the single proposed feature namely, joint total variation energy (JTVE) as a generalised feature for cross subject classification of a pair of mental tasks. The distinguishing ability of the proposed method is evaluated by three binary benchmark machine- learning classifiers namely, k-nearest neighbor (KNN), Support Vector Machine (SVM) and Decision Tree. Further, each signal is added with white noise to form noisy multi channel EEG signal, to validate the robustness of the proposed methodology using different SNRs. The simulation results demonstrate the effectiveness of the proposed method in presence of added noise with both the databases and outperforms the state of the art methods for mental task EEG classification.

The major contribution of the paper is summarized below.

- Address the mental task classification using Graph Signal representation of multi channel EEG signals.

- Functional connectivity of brain during each mental task is learned using Graph Signal Processing with smoothness constraint.

- Spectral feature of JTVE learned from smoothened graph of GSP technique is proposed which are robust to artifact contaminated EEG signal and added white Gaussian noise.

- Pair wise mental task cross subject classification within a database is performed by training the classifier using mixed features from multiple subjects and testing on unknown test subject.

The organization of the paper is as follows: Section II provides preliminaries followed by the description of the publicly available MTMC EEG databases. In section III the proposed methodology is presented in detail along with performance evaluation metrics. The simulation results and its analysis is presented in Section IV. Finally, section V concludes the paper with future scope of work.

\section{Preliminaries}

The following section describes basic preliminaries followed by the databases used.

Consider $M$ channel normalised EEG signal having time series of $N$ length, forming the channel wise data matrix $\mathbf{X} \in \mathbb{R}^{M \times N}=\left[\mathbf{x}_{1}, \ldots, \mathbf{x}_{i}, \ldots, \mathbf{x}_{M}\right]^{T}$ with amplitude of $\mathbf{x}_{i}$ restricted to a range of 0 to 1 . For graph signal representation of EEG signal $\mathbf{X}$, consider an undirected weighted graph defined as $\mathcal{G}=\{\mathcal{V}, \mathcal{E}, \mathcal{W}\}$ with each channel representing a vertex $v_{i} \in \mathcal{V}, \forall i \in\{1,2, \ldots, M\}$, corresponding to $\mathrm{N}$ length time series observations. Then, the graph signal $\mathbf{y}_{\mathbf{k}} \in \mathbb{R}^{M \times 1}$ is denoted by the column vector of matrix $\mathbf{X}$ with graph signal samples from the $k^{t h}$ time observation $\forall k \in\{1,2, \ldots, N\}$.

The weight matrix $\mathcal{W} \in \mathbb{R}_{+}^{m \times m}$ encodes the brain functional connectivity with $\mathbf{W}_{i, j}$ indicating the strength of the connection between nodes $\mathrm{i}$ and $\mathbf{j}$ and $\mathbf{W}_{i, i}=0$ for all $\mathrm{i}$. Spectral decomposition of weighted adjacency matrix $\mathbf{W}$ is defined as $\mathbf{W}=\mathbf{U} \Lambda \mathbf{U}^{T}$ where $\Lambda$ is the diagonal eigenvalues matrix with values $\lambda_{i}$ and its corresponding eigenvector $\mathbf{u}_{\mathbf{i}}, \forall 1 \leq i \leq M$ forming the eigenvector matrix $\mathbf{U}$. In addition, the degree matrix $\mathbf{D}$ is the diagonal matrix with $\mathbf{D}_{i, i}=\sum_{j} \mathbf{W}_{i, j}$. Then, the unnormalised graph Laplacian matrix $\mathbf{L}$ is given by $\mathbf{L}=\mathbf{D}-\mathbf{W}$ and spectral decomposition of $\mathbf{L}$ yields its eigenvalues $\omega_{i}, \forall 1 \leq i \leq M$.

\section{A. Mental Task based EEG Databases}

This section describes the mental task multi channel (MTMC) EEG databases used in this paper in detail.

- MTMC EEG Database I (Keirn and Aunon) For the classification of mental task EEG signals, publicly available mental task database by Keirn and Aunon [26] is used in this paper. The EEG data along with artifacts was recorded by placing 6 electrodes over C3, C4, P3, $\mathrm{P} 4, \mathrm{O} 1$, and $\mathrm{O} 2$ according to the $10-20$ international electrode placement systems. Data were recorded at a sampling rate of $250 \mathrm{~Hz}$ from seven subjects, however, subject 4 is not used due to incomplete information. EEG signals are recorded during each of the following five mental tasks performed by these subjects.

- Baseline Task (B): The subjects were asked to sit in relaxed state and no mental activity is performed.

- Multiplication Task (M): The subjects were given nontrivial multiplication problems, and were asked to solve them without vocalizing or making any other physical movements.

- Geometric Figure Rotation Task (R): The subjects were given 30 s to study a drawing of a complex three 
dimensional block figure after which the drawing was removed and the subjects were instructed to visualize the object being rotated about an axis.

- Letter Composing (L) : The subjects were asked to mentally compose a letter to a friend .

- Visual Counting Task (C): The subjects were asked to visualize numbers being written on the blackboard sequentially.

Each trial is recorded for $10 \mathrm{~s}$ with the sampling rate of 250 per second, which resulted in 2500 samples points per trial. The proposed method is tested for binary classification of all pairs of mental task states namely $\mathrm{B}$ vs $\mathrm{M}, \mathrm{B}$ vs $\mathrm{R}, \mathrm{B}$ vs $\mathrm{L}, \mathrm{B}$ vs $\mathrm{C}, \mathrm{M}$ vs $\mathrm{L}, \mathrm{M}$ vs $\mathrm{C}, \mathrm{M}$ vs R, L vs C, L vs R, and C vs R. Each EEG task signal with 5 trials is used in this paper.

- Mental Task EEG Database II (EEGMAT) Database II consists of public MIT Physionet EEG mental arithmetic task database [27], [28]. It contains artifact-free multichannel EEG recordings from 36 subjects. It consists of artifact-free EEG signals of 60s duration each with a sampling frequency of $500 \mathrm{~Hz}$. Only one trial with 19 EEG channel namely, FP1, FP2, F3, F4, Fz, F7, F8, C3, C4, Cz, P3, P4, Pz, O1, O2, T3, T4, T5, and T6 electrodes positioned according to the 10 - 20 international electrode placement system, was recorded per subject for each of the following task:

- Baseline Task (BT): The subjects did not perform any mental task and were asked to sit in a relaxed position

- Serial Subtraction Task (SS): Mental task involving Serial subtraction where subjects were instructed to perform a serial subtraction task including 4 digit minuend and 2 digit subtrahend and communicate the results orally.

The proposed method is evaluated for binary classification of mental task BT vs SS using initial $10 \mathrm{sec}$ of data from each signal.

\section{Methodology}

The proposed method is divided into various sections. Fig.1. shows the block diagram of proposed method for classification of a pair of mental task.

\section{A. Graph Learning from GSP perspective}

Learning a graph topology from the mental task based EEG signal deals with estimating the underlying relationship of the observed graph signals X. Functional relationship between different cortical regions changes corresponding to the changes in mental activity. Strength of the relationship between two regions $(i . j)$ is quantified by the edge weight $\left(w_{i, j}\right)$ of the learned graph. GSP perspective of graph learning is based on smooth graph signals on the learned graph. It implies that a graph signal is smooth if the signal values of well-connected (high edge weight) node pairs are similar [19]. The criteria for learning the graph is to minimize the signal variation i.e., minimize objective function $\operatorname{Tr}\left(\mathbf{X}^{T} \mathbf{L X}\right)$, which is defined as follows:

$$
\operatorname{Tr}\left(\mathbf{X}^{T} \mathbf{L X}\right)=\mathcal{Q}(\mathbf{L})=\frac{1}{2} \sum_{i, j} \mathbf{W}_{i, j}\left\|\left(\mathbf{x}_{i}-\mathbf{x}_{j}\right)\right\|^{2}
$$

where $\mathcal{Q}(\mathbf{L})$ is Laplacian quadratic form and $\mathbf{W}$ is the weight matrix representing functional connectivity.

By using weight matrix $\mathbf{W}_{i, j}$ and pairwise distance matrix $\mathbf{Z}_{i, j}=\left\|\left(\mathbf{x}_{i}-\mathbf{x}_{j}\right)\right\|^{2}, \mathbf{Z} \in \mathbb{R}^{m \times m}$, the trace term becomes $\operatorname{Tr}(\mathbf{W Z})$ which is equivalent to $\|(\mathbf{W} \circ \mathbf{Z})\|_{1,1}$. To infer the graph from EEG signal that favours the smoothness of the graph signals, following optimization problem [29] is formulated using this objective function with parameters $\alpha>0$ and $\beta \geq 0$ controlling the sparsity and positivity of the of the weight matrix.

$$
\min _{\mathbf{W}}\|(\mathbf{W} \circ \mathbf{Z})\|_{1,1}-\alpha \mathbf{1}^{(T)} \log (\mathbf{W} \mathbf{1})+\beta\|\mathbf{W}\|_{F}^{2}
$$

where, $\mathbf{1}=[1 \ldots, 1]^{T}$ and $\|(B)\|_{1,1}$ is the element wise norm- 1 of $B$, $\circ$ is the Hadamard product and $\|(.)\|_{F}$ is the Frobenius norm. Here, the smoothness term is a weighted $L-1$ norm of $\mathbf{W}$, which enforces sparsity and penalizes edges connecting distant rows of $\mathrm{X}$. The logarithmic barrier acting on the node degree vector (W1), forces the degrees to be positive. $\alpha$ and $\beta$ are scalars that control the scale and sparsity of the graph respectively. These values are empirically determined to maximise the learning performance for classification. When $\alpha$ increases with increase in $\beta$, we obtain a dense graph which results in poor classification, while decrease in $\beta$ with $\alpha$, produces sparse graph but few isolated nodes. Thus ratio of $\beta$ with $\alpha$ has to be maintained to ensure distinction between different mental tasks. Classification accuracy was checked for different combinations of $\alpha$ and $\beta$, the one giving the highest accuracy was chosen for the analysis. Using the above objective function the learned graph has a sparse set of edges when the given distances come from a smooth manifold, preferring only the ones associated with small distances in Z.

Thus, the estimated $\mathbf{W}_{m \times m}$ for graph $\mathcal{G}$ captures the connectivity of brain from given EEG signal corresponding to each mental task state.

\section{B. Extraction of Graph Feature Metric for mental task classification}

After building the graph $\mathcal{G}$ in the form of weighted adjacency matrix $W$ corresponding to each mental task, following graph metrics are proposed to extract the distinctive features which can classify different mental task EEG signals.

\section{- Two norm total variation of eigen vector (TNTV) :} To quantify the interaction between graph signal and corresponding graph structure, the difference between the graph signal and its first graph shift is defined as signal change $\triangle \mathbf{y}=\mathbf{y}-\mathbf{y}_{1}=\mathbf{y}-\mathbf{W}_{\text {norm }} \mathbf{y}$ where $\mathbf{W}_{\text {norm }}$ is normalised weighted adjacency matrix [25]. The energy of this signal change defined as $\mathbf{E}_{\triangle y}=$ $\left\|\mathbf{y}-\mathbf{W}_{\text {norm }} \mathbf{y}\right\|_{2}^{2}$, which is indicative of frequency variation in terms of the rate of eigenvector change along time. When graph signal $\mathbf{y}$ assumes a specific eigenvector $\mathbf{u}$, 


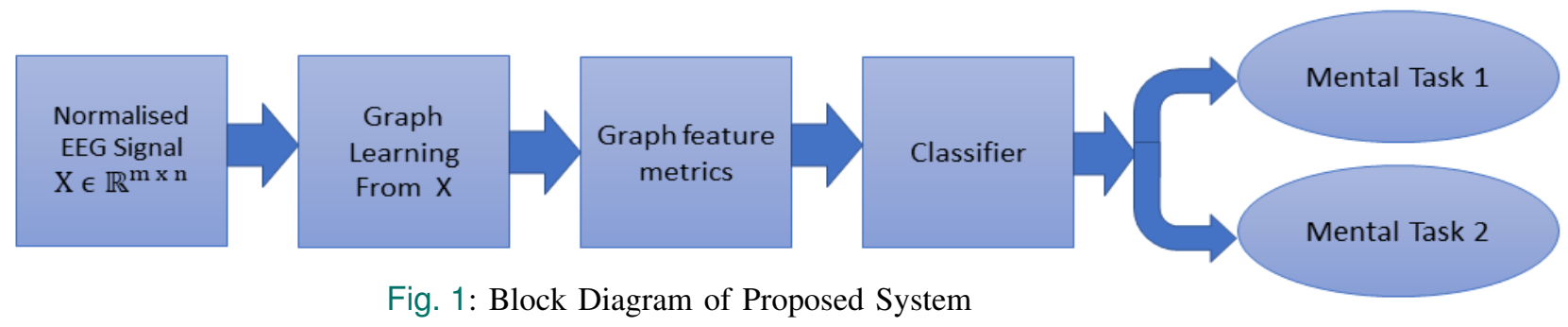

the energy of this eigenvector change is referred as twonorm total variation of an eigen vector given by

$$
\mathbf{E}_{\triangle \mathbf{u}}=\left\|\mathbf{u}-\frac{\lambda \mathbf{u}}{\lambda_{\max }}\right\|_{2}^{2}=\left\|1-\frac{\lambda}{\lambda_{\max }}\right\|^{2}
$$

which satisfies the relation $\mathbf{W u}=\lambda \mathbf{u}$ and $\|\mathbf{u}\|^{2}=1$. Thus, low energy change in eigen vector $\mathbf{u}$ corresponds to low spectral content of the graph signal i.e lower values of $\mathbf{E}_{\triangle \mathbf{u}}$ are associated with slow varying changes of $\mathbf{u}$ in time. This energy change is minimal for $\lambda=$ $\lambda_{\max }$ and increases with decrease in $\lambda$ of the weighted adjacency matrix. In this paper, the average of the $\mathbf{E}_{\triangle \mathbf{u}}$ corresponding to only the low frequency components $\left(\lambda_{i}\right.$ for $M / 3 \leq i \leq M)$ is used as a feature for classification.

$$
\operatorname{TNTV}(\mathcal{G})=\widetilde{\mathbf{E}_{\triangle \mathbf{u}}}
$$

- Graph Laplacian Energy (GLE) GLE is a measure to compute graph energy which is an important graph characteristic used to quantify the graph complexity [30]. Graph Energy using eigenvalues of the Laplacian matrix defined in terms of Laplacian Energy (LE) is given by

$$
L E(G)=\sum_{i=1}^{M}\left|\omega_{i}-\widetilde{\mathbf{D}}\right|
$$

where $\omega$ are the eigenvalues of the Laplacian matrix of graph and $\widetilde{\mathbf{D}}$ is average node degree defined as $\widetilde{\mathbf{D}}=$ $2 \mathbf{E} / \mathbf{M}$, with $E$ is number of edges and $M$ being the number of nodes of graph. The mean of LE for each graph signal defined over $\mathcal{G}$ is used as a feature for classification.

$$
G L E(\mathcal{G})=\widetilde{L E_{G}}
$$

- Joint Total variation Energy (JTVE) : A new combined feature is proposed which takes into account graph energy in terms of GLE and eigen vector energy change in terms of TNTV. This measure is a convex sum of TNTV and GLE defined as follows.

$$
J T V E\left(\mathcal{G}_{i}, \mathcal{G}_{j}\right)=\rho \times T N T V+(1-\rho) G L E
$$

where $\rho \subseteq[0,1]$ is a weighted parameter controlling the contributions of TNTV and GLE. Using (4) , (6) and (7), all three features are calculated from the graph representations $G$ to serve as metric for classifiers. To assess the significance of these features, Kruskal Wallis statistical test [31] for all possible pairs of mental tasks is performed, whose results are tabulated in Table I. It is observed that the combined convex sum measure JTVE is sufficient single feature for classification as it has the desired least $\mathrm{p}$ value among all three features. Further, feature values of GLE, TNTV and JTVE for baseline task (BL) and serial subtraction task (SS) from the Database II, is shown in Fig. 2 where the ability of the single JTVE to distinguish the pair of mental tasks is observed. Thus single convex sum feature is finally fed to the classifier to classify different pairs of mental task EEG signals.

TABLE I:

p-values for feature metrics using Kruskal-Wallis test

\begin{tabular}{|c|c|c|c|}
\hline \multicolumn{5}{|c|}{ Database I: (Keirn and Aunon) } \\
\hline Test Case & TNTV & GLE & JTVE \\
\hline B vs M & 0.0166 & 0.0052 & $2.8 \times 10^{-} 11$ \\
\hline B vs C & 0.003 & 0.009 & $3.5 \times 10^{-} 11$ \\
\hline B vs L & 0.006 & 0.0027 & $2.8 \times 10^{-} 11$ \\
\hline B vs R & 0.0003 & 0.0153 & $2.8 \times 10^{-} 11$ \\
\hline M vs C & 0.019 & 0.01 & $2.1 \times 10^{-} 11$ \\
\hline M vs L & 0.01 & 0.05 & $1.8 \times 10^{-} 11$ \\
\hline M vs R & 0.07 & 0.01 & $2.5 \times 10^{-} 11$ \\
\hline C vs L & 0.07 & 0.05 & $2 \times 10^{-} 11$ \\
\hline C vs R & 0.06 & 0.07 & $2 \times 10^{-} 11$ \\
\hline L vs R & 0.06 & 0.04 & $2 \times 10^{-} 11$ \\
\hline \multicolumn{5}{|c|}{ Database II: (EEGMAT) } \\
\hline BL vs SS & 0.0003 & 0.005 & $1.9 \times 10^{-} 11$ \\
\hline
\end{tabular}
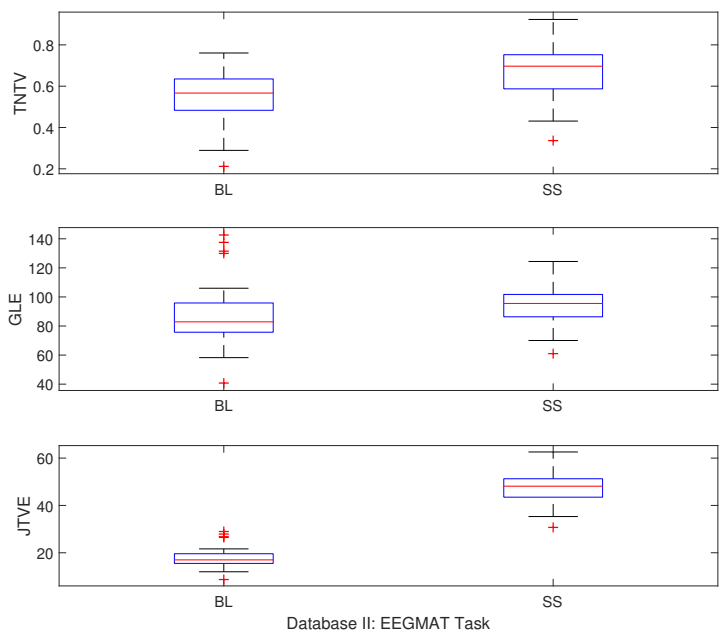

Fig. 2: Boxplot of feature metrics for BL vs SS task pair

\section{Classifier}

The convex sum feature of JTVE extracted from graphs of mental task pair of EEG signals is fed to the three different binary benchmark classifiers namely K Nearest Neighbour 
(KNN) [9], SVM classifier [2] and Decision Tree classifier [32]. For evaluating the effectiveness of the proposed methodology under subject invariant conditions [33], training of the classifier for a pair of mental task EEG signals is performed only once with mixed JTVE feature from all subjects within a database. Thus, the system gets pre-trained by the features extracted from pool of subjects and gets tested by unknown test subject [34], which increases the efficacy for BCI application. 10 fold cross validation is used to validate the proposed method where for each database, cross-subject feature from a pair of mental task is divided into 10 equal sized partitions. Subsequently, 10 iterations of training and testing is performed such that for each iteration, a distinct fold of feature data is held out for testing and remaining 9 folds are used for training.

\section{Performance Evaluation Metrics}

- Confusion Matrix: The performance of the proposed method using benchmark classifiers are presented using confusion matrices, which is tabular way of presenting the performance of the classifiers as shown in Fig. 4. For binary classification of each task pair, for example, BL vs SS, this paper considers positive class as mental baseline task (BL) and negative as mental serial subtraction task (SS). Similar interpretation is followed in other task pairs correspondingly.

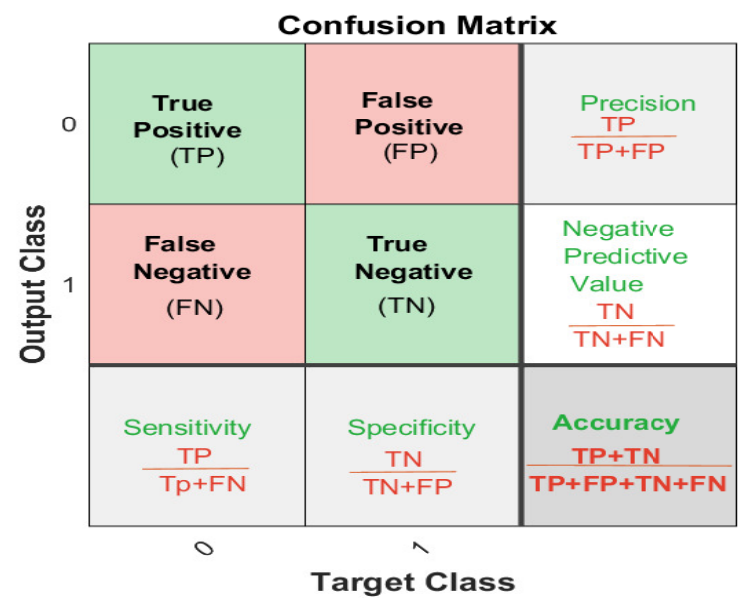

Fig. 3: Details of Confusion Matrix

In addition to the metrics defined in confusion matrix, FScore giving harmonic mean of sensitivity and precision is also computed to evaluate the proposed method.

$$
\mathrm{F}-\mathrm{Score}=\frac{2(\mathrm{TP})}{2(\mathrm{TP})+(\mathrm{FP})+(\mathrm{FN})}
$$

- Information transfer rate (ITR) Information Transfer Rate (ITR) [35] is a standard measure of performance used in BCI applications expressed in bits per minute(bpm) It combines the accuracy and the speed of the classifier into a single number which shows how much information is transferred by the $\mathrm{BCI}$ in one unit of time, defined as follows:

$$
\operatorname{ITR}(\mathrm{bpm})=\frac{\mathrm{B}}{\mathrm{T}}
$$

$$
\mathrm{B}=\log _{2} Q+\mathrm{P} \log _{2} \mathrm{P}+(1-\mathrm{P}) \log _{2} \frac{1-\mathrm{P}}{\mathrm{Q}-1}
$$

where $\mathrm{B}$ is ITR in bit rate(bits/symbol). Q, P and T stand for number of classes, accuracy, and computational time respectively. Computational time indicate time required to learn the graph from a given multi channel EEG signal using GSP method and extracting convex sum feature of JTVE. The whole process of computation is performed using Intel(R) evo Core(TM) i7-processor with 4.7 $\mathrm{GHz}$ clock speed and $16 \mathrm{~GB}$ RAM and the algorithm implemented on MATLAB R2021b 64-bit version.

\section{Results and Discussion}

- Analysis of each feature separately: To evaluate the effectiveness of the proposed feature TVEG, GLE, and JTVE is used alone as a feature for obtaining the classification. Fig. 4 shows the cross subject classification accuracy achieved for each task pair corresponding to each feature on both the databases. It is observed that the single feature of convex sum based JTVG is sufficient for classification.

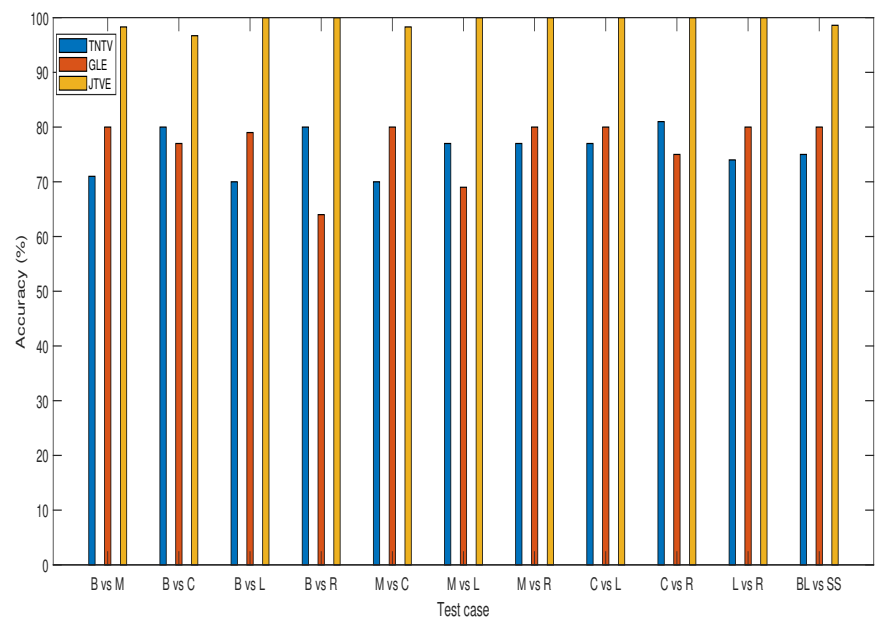

Fig. 4: Accuracy with the each proposed feature alone

- Effect of $\rho$ in JTVE: To further check the performance of the proposed feature of JTVE, Fig. 5 shows the average accuracy with different values of $\rho$. From the analysis, it is shown that for $0.2 \leq \rho \leq 0.6$, JTVE performs universally the same. For this paper, we have fixed $\rho=$ 0.4 having the highest classification performance.

- Analysis on Database I and II: Performance of the proposed methodology on artifact free database II (EEGMAT) with all the three classifiers is shown in Fig 6. Similar performance is achieved with all three benchmark classifiers with the best classification accuracy of $98.6 \%$ with both SVM and KNN classifiers.

The results of the proposed method with Database I is presented in Fig.8, Fig.9 and Fig.10. It tabulates the confusion matrices for classification of different mental task pairs of database I (Keirn and Aunon) using benchmark classifiers of SVM, KNN and DT respectively. It is observed that the proposed method shows 


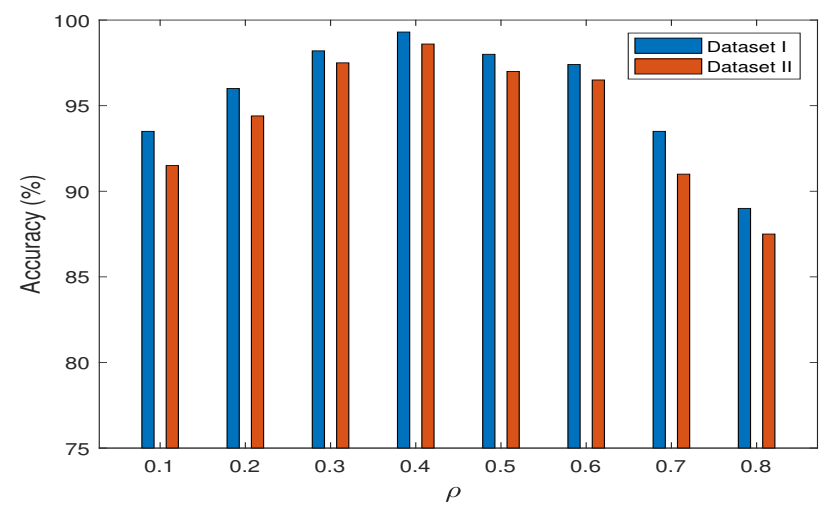

Fig. 5: Average Accuracy with different values of $\rho$

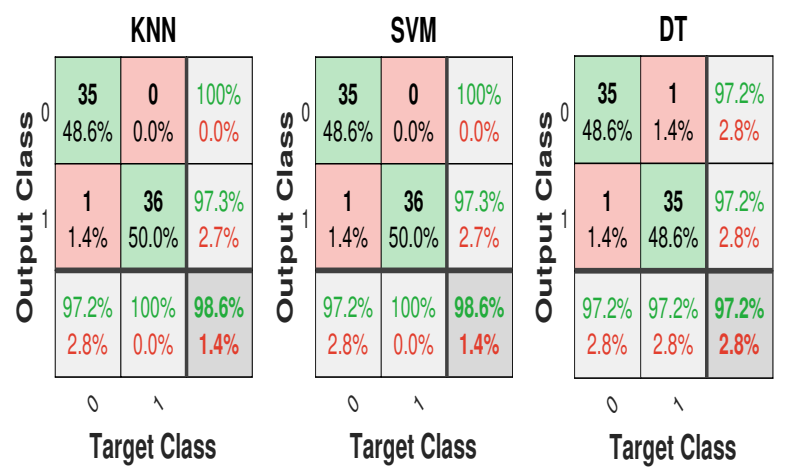

Fig. 6: Confusion Matrices for Database II: EEGMAT

similar performance with all the classifiers and achieves best average accuracy of $99.33 \%$ using SVM classifier. Thus functional connectivity based proposed method can differentiate different mental task pairs, without using an artifact suppression or removal step for artifact contaminated database I.

- Comparison with existing literature: To compare the proposed method with state of the art techniques, Table II tabulates the average accuracy of the proposed method obtained using SVM classifier, with existing techniques reported in literature. It can be observed method outperforms the existing work for both the databases.

- Analysis using conventional graph learning methods: The superiority of the proposed metric obtained from the smoothened graph of GSP technique is validated by comparing it with Pearson correlation [36] and Gaussian radial basis function (RBF) [22] based functional connectivity as shown in Table III. An important benefit of the proposed method is the natural sparsity induced by the regularization design. The average computational time for correlation and RBF based method is $0.7 \mathrm{sec}$ while GSP requires $0.92 \mathrm{sec}$. Table III shows the superiority of the purposed GSP based JTVE feature in terms of classification accuracy, F score and ITR (bpm) using SVM classifier.

- Effect of adding white Gaussian noise: To further validate the robustness of the proposed method against noise, the MTMC EEG signals are added with white Gaussian noise with different SNRs. Fig. 7 shows the average accuracy of the method on AWGN added database I and
TABLE II:

Comparative analysis of the average classification accuracy of proposed methodology with existing work.

\begin{tabular}{|c|c|c|}
\hline \multicolumn{3}{|c|}{ Dataset I:Keirn and Aunon Database } \\
\hline Author & \begin{tabular}{|c|} 
Technique \\
\end{tabular} & Accuracy (\%) \\
\hline R. Palaniappan [4] & Gamma Band Features & 73.28 \\
\hline Li Zhang al. [6] & High Frequency Band Information & 76.4 \\
\hline H. U. Amin. [9] & Wavelet Decomposition & 97.86 \\
\hline A. Gupta et.al. [1] & Power Spectral Density & 85 \\
\hline S. Noshadi et.al. [16] & Empirical Mode Decomposition & 97 \\
\hline S. Dutta et. al. [8] & Multivariate EMD & 94 \\
\hline Z.A.A Alyasseri et.al. [7] & Wavelet Decomposition & 87 \\
\hline Proposed & Graph Signal Processing & 99.3 \\
\hline \multicolumn{3}{|c|}{ Dataset II:EEGMAT Database } \\
\hline M. Saini et. al. [12] & Variational Mode Decomposition & 93 \\
\hline B. Ganguly et. al. [14] & LSTM Network & 91.67 \\
\hline B. Fatimah et. al. [5] & Fourier Decomposition & 98.6 \\
\hline A. Varshney et.al. [3] & Recurrent Neural Network & 99.38 \\
\hline Proposed & Graph Signal Processing & 98.6 \\
\hline
\end{tabular}

TABLE III:

Performance comparison of proposed methodology using conventional graph learning methods

\begin{tabular}{|c|c|c|c|c|c|c|}
\hline \multirow{2}{*}{ Graph } & \multicolumn{2}{|c|}{ Accuracy } & \multicolumn{2}{c|}{ F-Score } & \multicolumn{2}{c|}{ ITR (bpm) } \\
\cline { 2 - 7 } & Dataset & Dataset & Dataset & Dataset & Dataset & Dataset \\
I & II & I & II & I & II \\
\hline GSP & 99.3 & 98.6 & 99.36 & 98.5 & 66.33 & 53.62 \\
\hline RBF & 91 & 88.7 & 90 & 87.4 & 61.25 & 32.74 \\
\hline Pearson Correlation & 94.5 & 93 & 93.9 & 91.5 & 63.2 & 54.34 \\
\hline
\end{tabular}

II with different SNRs. It can be seen from the figure that the classification is fairly the same even in the presence of this noise in both the cases. Whereas the performance using RBF and correlation based functional connectivity is degraded with different SNRs as shown in Fig. 7.

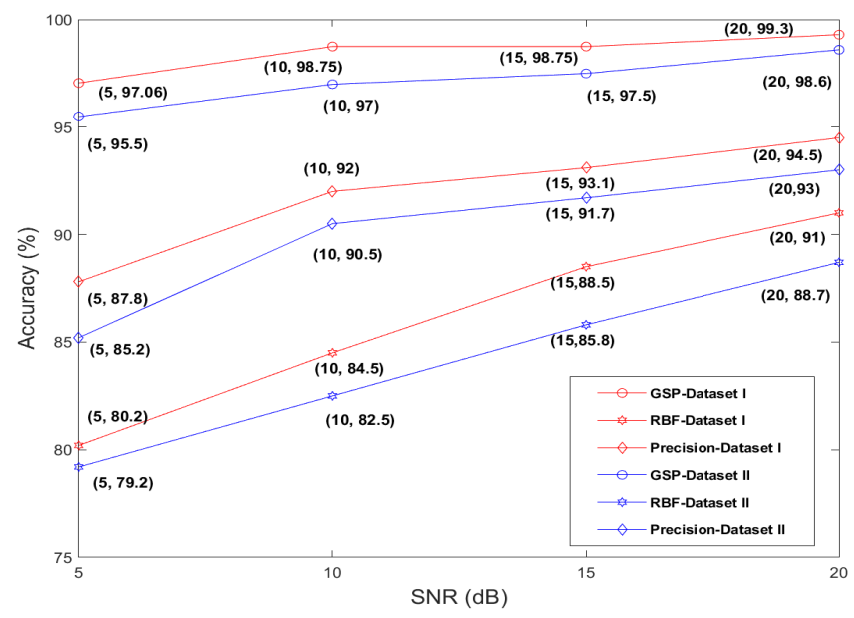

Fig. 7: Average Performance with AWGN

\section{CONCLUSion And Future Scope}

This paper presented a novel technique of smooth graph signal representation of multi channel EEG signal for mental task classification. The proposed work shows that the single 
graph spectral feature called JTVE can differentiate the different mental task pairs with noise and artifact free database II. It also performs equally well in artifact contaminated database I. Further, the performance of the classification remains fairly the same in additive white noise conditions with the proposed JTVE metric. The future avenue lies in examining vertex spectral representation of GSP to further characterize the mental task EEG signal to provide new insights about localizing the brain's topological regions during mental activity. Further, extending the binary mental-task classification to multi-class is one of the future scope followed by real-time classification of mental task from EEG signals for BCI environment.

\section{REFERENCES}

[1] A. Gupta, R. K. Agrawal, J. S. Kirar, J. Andreu-Perez, W.-P. Ding, C.-T Lin, and M. Prasad, "On the utility of power spectral techniques with feature selection techniques for effective mental task classification in noninvasive BCI," IEEE Transactions on Systems, Man, and Cybernetics: Systems, 2019.

[2] N. N. Neshov, A. H. Manolova, I. R. Draganov, K. T. Tonschev, and O. L. Boumbarov, "Classification of mental tasks from EEG signals using spectral analysis, PCA and SVM," Cybernetics and Information Technologies, vol. 18, no. 1, pp. 81-92, 2018.

[3] A. Varshney, S. K. Ghosh, S. Padhy, R. K. Tripathy, and U. R. Acharya, "Automated classification of mental arithmetic tasks using recurrent neural network and entropy features obtained from multi-channel EEG signals," Electronics, vol. 10, no. 9, p. 1079, 2021.

[4] R. Palaniappan, "Utilizing gamma band to improve mental task based brain-computer interface design," IEEE Transactions on Neural Systems and Rehabilitation Engineering, vol. 14, no. 3, pp. 299-303, 2006.

[5] B. Fatimah, A. Javali, H. Ansar, B. Harshitha, and H. Kumar, "Mental Arithmetic Task Classification using Fourier Decomposition Method," in 2020 International Conference on Communication and Signal Processing (ICCSP), pp. 0046-0050, IEEE, 2020.

[6] L. Zhang, W. He, C. He, and P. Wang, "Improving mental task classification by adding high frequency band information," Journal of Medical Systems, vol. 34, no. 1, pp. 51-60, 2010.

[7] Z. A. A. Alyasseri, A. T. Khadeer, M. A. Al-Betar, A. Abasi, S. Makhadmeh, and N. S. Ali, "The effects of EEG feature extraction using multiwavelet decomposition for mental tasks classification," in Proceedings of the International Conference on Information and Communication Technology, pp. 139-146, 2019.

[8] S. Dutta, M. Singh, and A. Kumar, "Automated classification of nonmotor mental task in electroencephalogram based brain-computer interface using multivariate autoregressive model in the intrinsic mode function domain," Biomedical Signal Processing and Control, vol. 43, pp. 174-182, 2018 .

[9] H. U. Amin, W. Mumtaz, A. R. Subhani, M. N. M. Saad, and A. S. Malik, "Classification of EEG signals based on pattern recognition approach," Frontiers in Computational Neuroscience, vol. 11, p. 103, 2017.

[10] M. T. Sadiq, X. Yu, Z. Yuan, F. Zeming, A. U. Rehman, I. Ullah, G. Li, and G. Xiao, "Motor Imagery EEG Signals Decoding by Multivariate Empirical Wavelet Transform-Based Framework for Robust BrainCcomputer Interfaces," IEEE access, vol. 7, pp. 171431-171451, 2019.

[11] M. T. Sadiq, X. Yu, Z. Yuan, Z. Fan, A. U. Rehman, G. Li, and G. Xiao, "Motor imagery EEG signals classification based on mode amplitude and frequency components using empirical wavelet transform," IEEE Access, vol. 7, pp. 127678-127692, 2019.

[12] M. Saini, U. Satija, and M. D. Upadhayay, "Variational mode decomposition based mental task classification from electroencephalogram," in 2020 IEEE 17th India Council International Conference (INDICON), pp. 1-7, IEEE, 2020.

[13] M. T. Sadiq, X. Yu, Z. Yuan, and M. Z. Aziz, "Motor Imagery BCI Classification Based on Novel Two-Dimensional Modelling in Empirical Wavelet Transform," Electron. Lett, vol. 56, no. 25, pp. 1367-1369, 2020.

[14] B. Ganguly, A. Chatterjee, W. Mehdi, S. Sharma, and S. Garai, "EEG Based Mental Arithmetic Task Classification Using a Stacked Long Short Term Memory Network for Brain-Computer Interfacing," in 2020 IEEE VLSI DEVICE CIRCUIT AND SYSTEM (VLSI DCS), pp. 89-94, IEEE, 2020
[15] A. Craik, Y. He, and J. L. Contreras-Vidal, "Deep learning for electroencephalogram (EEG) classification tasks: a review," Journal of Neural Engineering, vol. 16, no. 3, p. 031001, 2019.

[16] S. Noshadi, V. Abootalebi, M. T. Sadeghi, and M. S. Shahvazian, "Selection of an efficient feature space for EEG-based mental task discrimination," Biocybernetics and Biomedical Engineering, vol. 34, no. 3, pp. 159-168, 2014

[17] M. T. Sadiq, X. Yu, and Z. Yuan, "Exploiting Dimensionality Reduction and Neural Network Techniques for the Development of Expert Brain-Computer Interfaces," Expert Systems with Applications, vol. 164, p. 114031, 2021.

[18] C. Hu, L. Cheng, J. Sepulcre, K. A. Johnson, G. E. Fakhri, Y. M. Lu, and Q. Li, "A spectral graph regression model for learning brain connectivity of Alzheimer's disease," PLOS One, vol. 10, no. 5, p. e0128136, 2015.

[19] S. Gao, X. Xia, D. Scheinost, and G. Mishne, "Smooth graph learning for functional connectivity estimation," NeuroImage, vol. 239, p. 118289 , 2021.

[20] G. N. Dimitrakopoulos, I. Kakkos, Z. Dai, J. Lim, J. J. deSouza, A. Bezerianos, and Y. Sun, "Task-independent mental workload classification based upon common multiband EEG cortical connectivity," IEEE Transactions on Neural Systems and Rehabilitation Engineering, vol. 25, no. 11, pp. 1940-1949, 2017.

[21] L. Rui, H. Nejati, and N.-M. Cheung, "Dimensionality reduction of brain imaging data using graph signal processing," in 2016 IEEE International Conference on Image Processing (ICIP), pp. 1329-1333, IEEE, 2016.

[22] X. Dong, D. Thanou, M. Rabbat, and P. Frossard, "Learning graphs from data: A signal representation perspective," IEEE Signal Processing Magazine, vol. 36, no. 3, pp. 44-63, 2019.

[23] X. Dong, D. Thanou, P. Frossard, and P. Vandergheynst, "Learning laplacian matrix in smooth graph signal representations," IEEE Transactions on Signal Processing, vol. 64, no. 23, pp. 6160-6173, 2016.

[24] S. S. Saboksayr, G. Mateos, and M. Cetin, "EEG-based emotion classification using graph signal processing," in ICASSP 2021-2021 IEEE International Conference on Acoustics, Speech and Signal Processing (ICASSP), pp. 1065-1069, IEEE, 2021.

[25] L. Stanković, D. Mandic, M. Daković, M. Brajović, B. Scalzo, S. Li, and A. G. Constantinides, "Data analytics on graphs part II: Signals on graphs," Foundations and Trends® in Machine Learning, vol. 13, no. 2-3, pp. 158-331, 2020.

[26] Z. A. Keirn and J. I. Aunon, "A new mode of communication between man and his surroundings," IEEE transactions on biomedical engineering, vol. 37, no. 12, pp. 1209-1214, 1990.

[27] I. Zyma, S. Tukaev, I. Seleznov, K. Kiyono, A. Popov, M. Chernykh, and O. Shpenkov, "Electroencephalograms during mental arithmetic task performance," Data, vol. 4, no. 1, p. 14, 2019.

[28] A. L. Goldberger, L. A. Amaral, L. Glass, J. M. Hausdorff, P. C. Ivanov, R. G. Mark, J. E. Mietus, G. B. Moody, C.-K. Peng, and H. E. Stanley, "Physiobank, physiotoolkit, and physionet: components of a new research resource for complex physiologic signals," circulation, vol. 101 , no. 23 , pp. e215-e220, 2000

[29] V. Kalofolias, "How to learn a graph from smooth signals," in Artificial Intelligence and Statistics, pp. 920-929, 2016.

[30] H. B. Ahmed, D. Dare, and A.-O. Boudraa, "Graph signals classification using total variation and graph energy informations," in 2017 IEEE Global Conference on Signal and Information Processing (GlobalSIP), pp. 667-671, IEEE, 2017.

[31] E. Ostertagova, O. Ostertag, and J. Kováč, "Methodology and application of the kruskal-wallis test," Applied Mechanics and Materials, vol. 611, pp. 115-120, 082014.

[32] V. Kotu and B. Deshpande, "Predictive Analytics and Data Mining: Concepts and Practice with Rapidminer". Morgan Kaufmann, 2014.

[33] Z. Wang, R. M. Hope, Z. Wang, Q. Ji, and W. D. Gray, "Cross-subject workload classification with a hierarchical bayes model," NeuroImage, vol. 59, no. 1, pp. 64-69, 2012.

[34] Z. Yin, Y. Wang, L. Liu, W. Zhang, and J. Zhang, "Cross-Subject EEG Feature Selection for Emotion Recognition using Transfer Recursive Feature Elimination," Frontiers in Neurorobotics, vol. 11, p. 19, 2017.

[35] M. Shahbakhti, M. Beiramvand, I. Rejer, P. Augustyniak, A. BroniecWojcik, M. Wierzchon, and V. Marozas, "Simultaneous eye blink characterization and elimination from low-channel prefrontal eeg signals enhances driver drowsiness detection," IEEE Journal of Biomedical and Health Informatics, 2021.

[36] R. Liégeois, A. Santos, V. Matta, D. Van De Ville, and A. H. Sayed, "Revisiting correlation-based functional connectivity and its relationship with structural connectivity," Network Neuroscience, vol. 4, no. 4, pp. 1235-1251, 2020. 


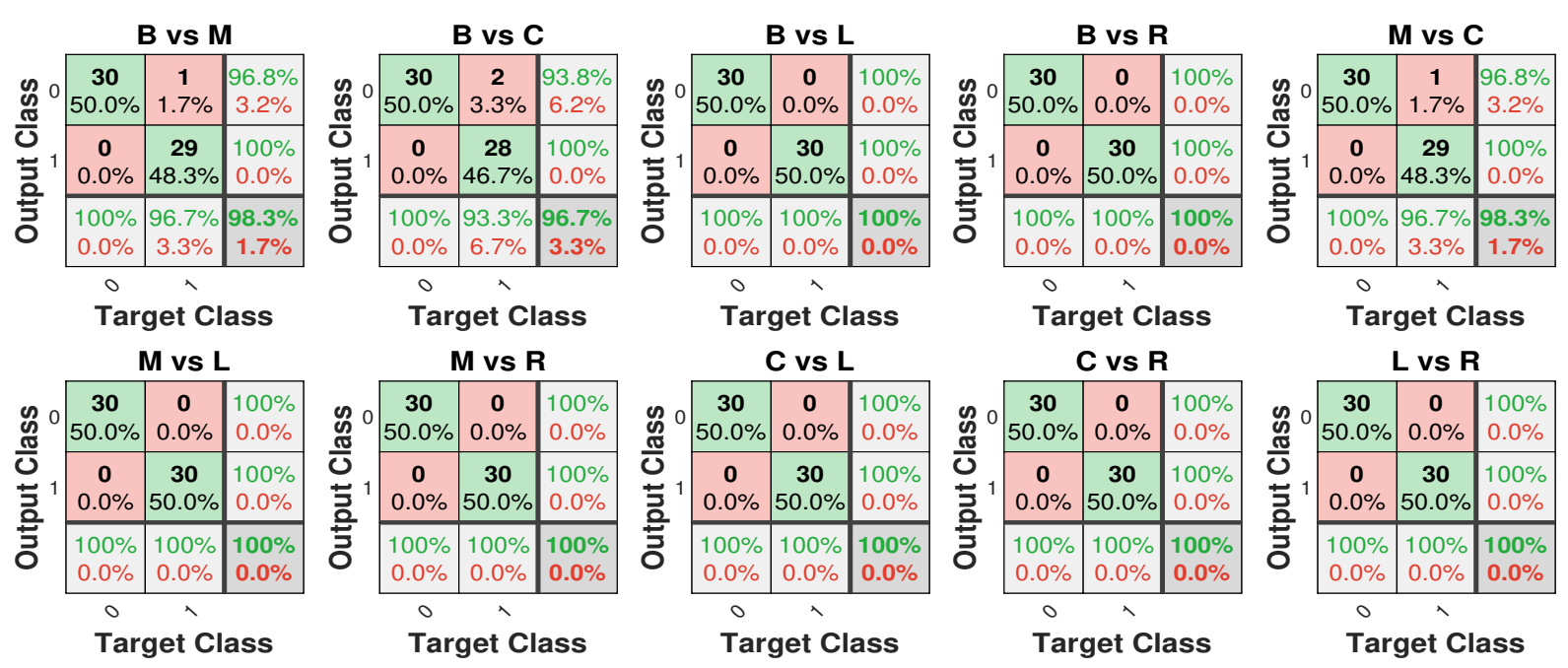

Fig. 8: Confusion Matrices for different test cases of Keirn and Aunon Database using SVM classifier
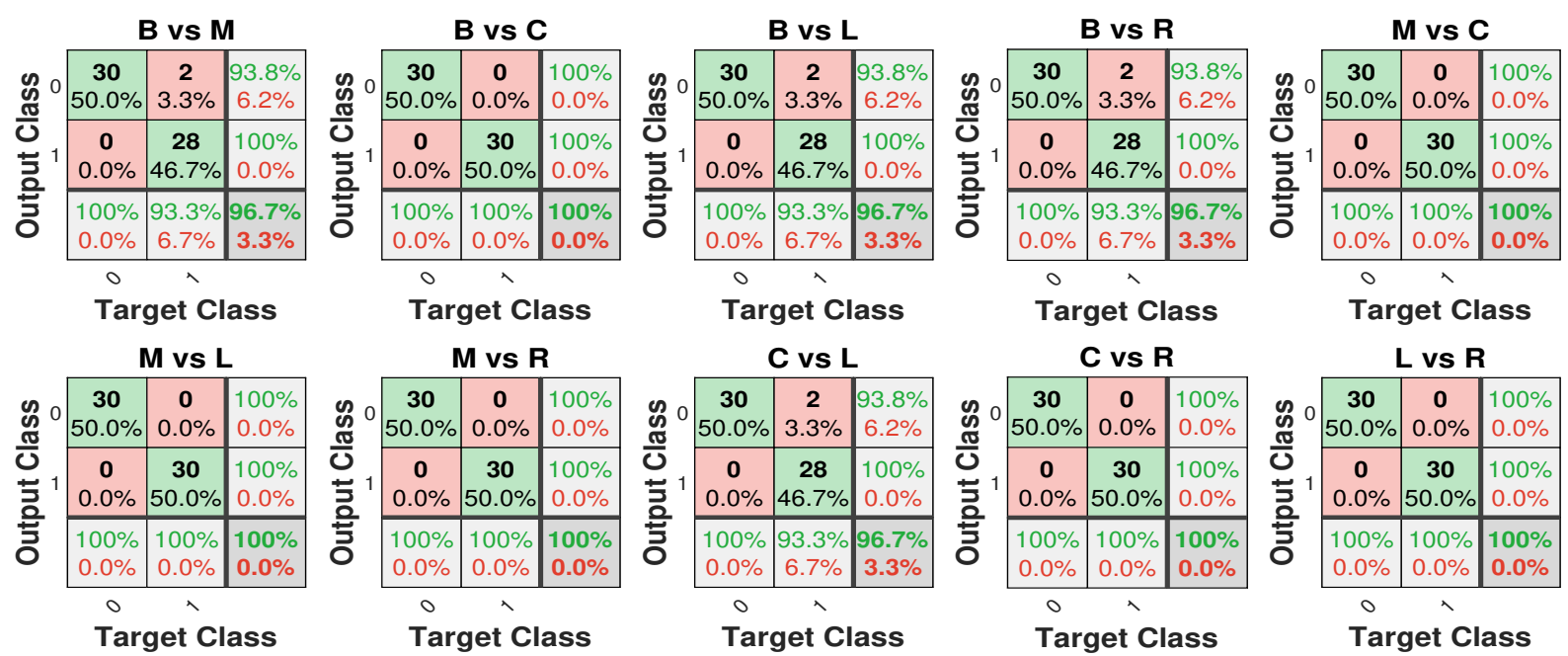

Fig. 9: Confusion Matrices for different test cases of Keirn and Aunon Database using KNN classifier
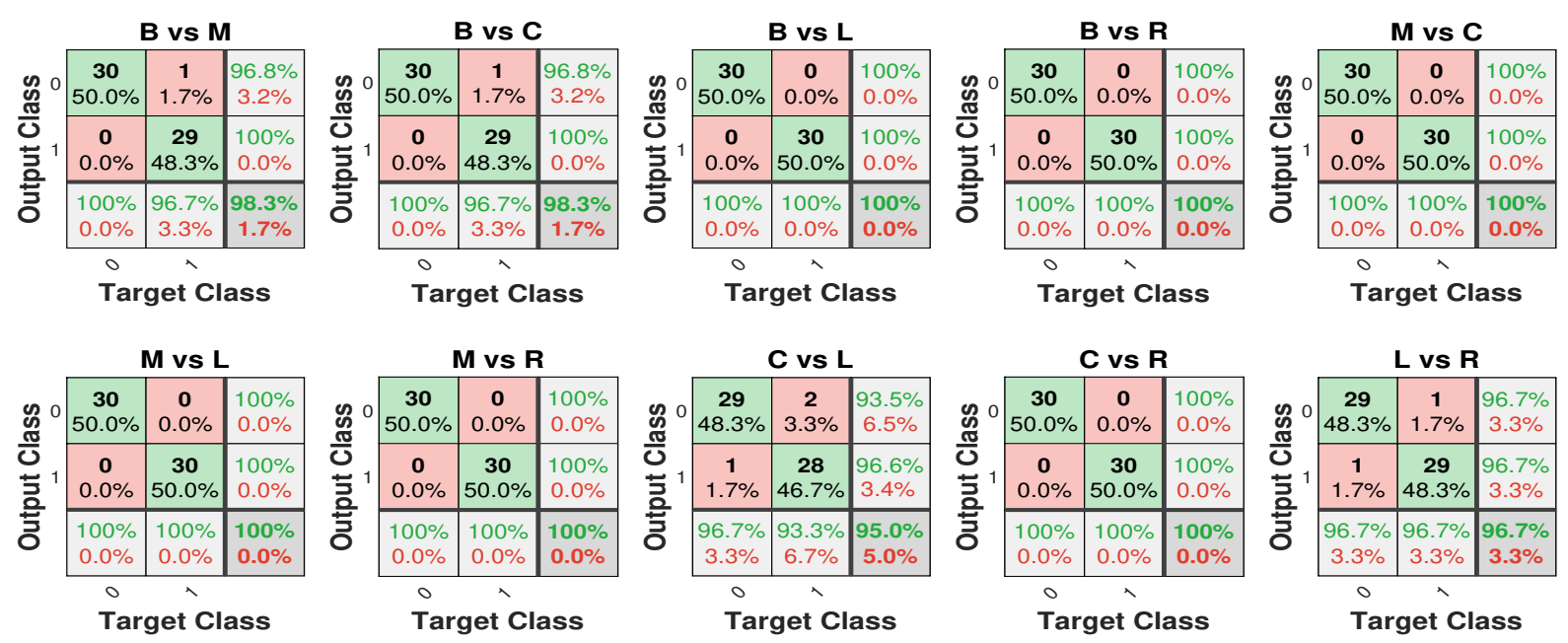

Fig. 10: Confusion Matrices for different test cases of Keirn and Aunon Database using Decision Tree classifier 